\title{
Criminologie
}

\section{Document : Une requête un peu particulière}

Volume 18, numéro 1, 1985

L’histoire du contrôle social

URI : https://id.erudit.org/iderudit/017204ar

DOI : https://doi.org/10.7202/017204ar

Aller au sommaire du numéro

Éditeur(s)

Les Presses de l'Université de Montréal

ISSN

0316-0041 (imprimé)

1492-1367 (numérique)

Découvrir la revue

Citer ce document

(1985). Document : Une requête un peu particulière. Criminologie, 18(1), 19-24.

https://doi.org/10.7202/017204ar d'utilisation que vous pouvez consulter en ligne.

https://apropos.erudit.org/fr/usagers/politique-dutilisation/ 


\section{UNE REQUÊTE UN PEU PARTICULIÈRE}

En 1708, Michel Taillon, un habitant de l'île Jésus, se plaint d'avoir été victime de voies de fait de la part de personnes de la Pointe-auxTrembles, les Beauchamp. Ceux-ci soutiennent que l'origine du conflit tiendrait au fait que Taillon aurait injurié des femmes de la Pointe-auxTrembles. Le curé Roche de cette paroisse intervient en adressant au juge royal, la requête qui suit. Il peut être intéressant de noter que, peu après, l'action pénale fut transformée en enquête civile. 
20

CRIMINOLOGIE

Ims

$+$

- Lapointer aun trombles les 23 jiniz nor.

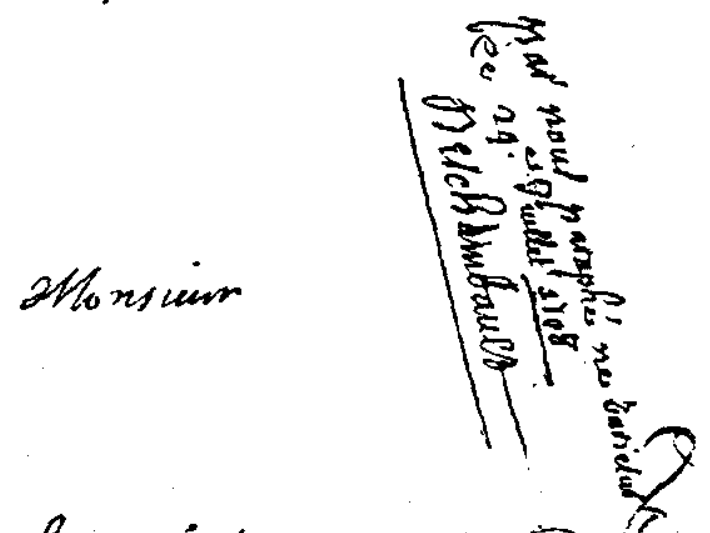

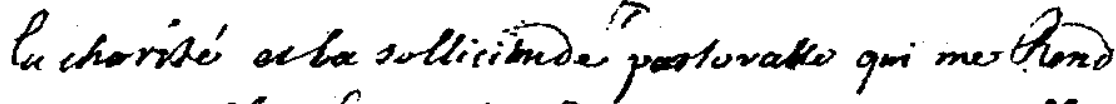
wes Sevisbles Les affaires de mes paositiens mobligant

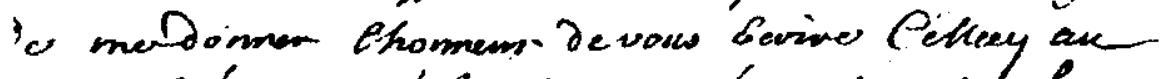

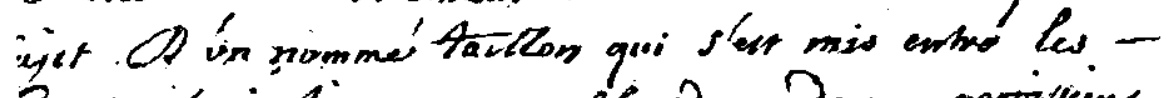

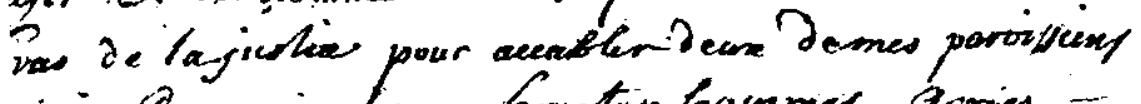

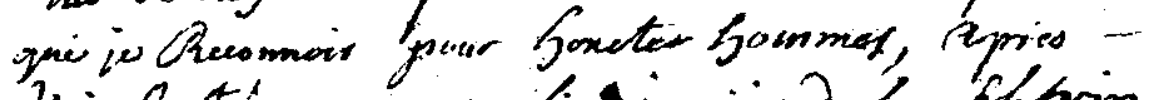

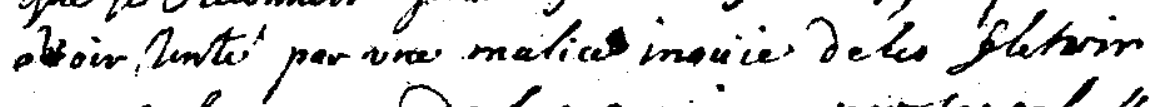
prov sa langues, ond des oppirimer pow ses cabaltes

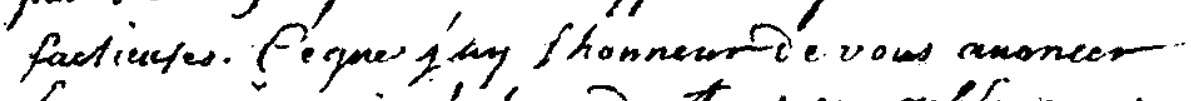
La, monfum, je terhos. de tout un publegryal un' de ce fout erdes environt ne ma' encormie.' 
LE CONTRÔle SOCLAL DANS LA SOCIÉTÉ CANADIENNE

21

$+$

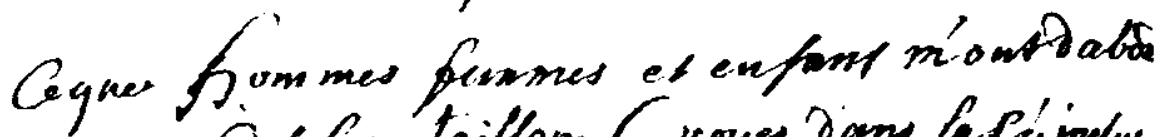

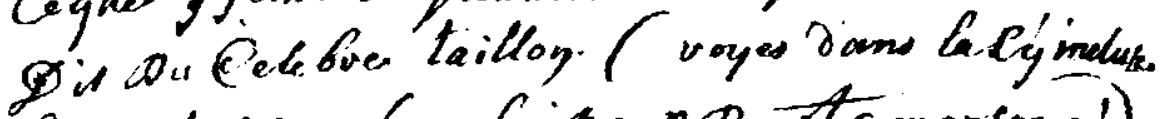

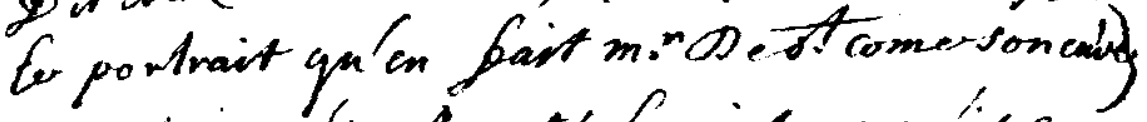

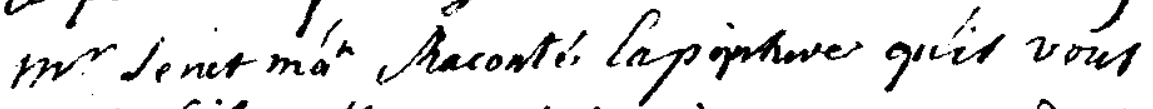

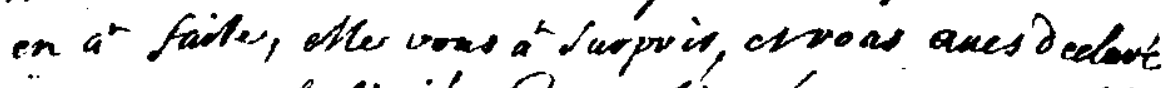
que vous chatierice (emortion s'et vous a prorto if

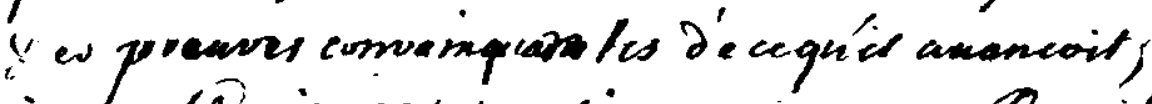
je. hes (evois pas montieno/g gues vour Recusiés

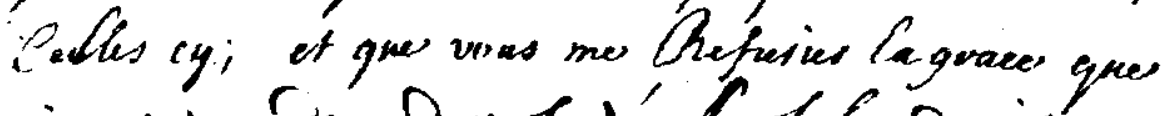

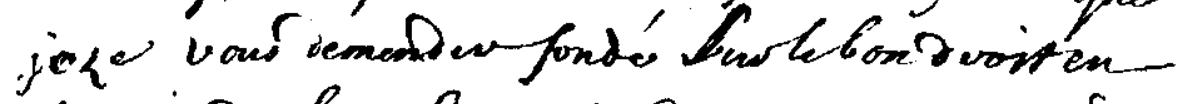
fanewr D el beane Sampot: Peser oit covommer bor

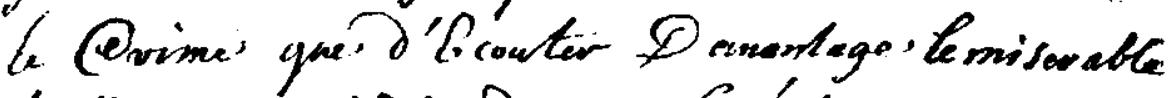

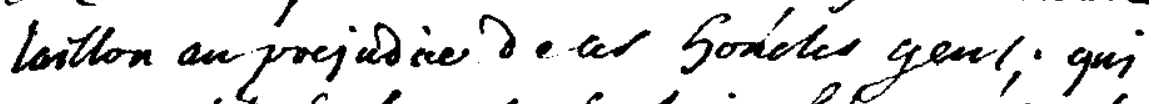

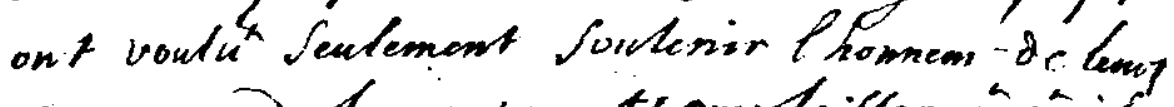

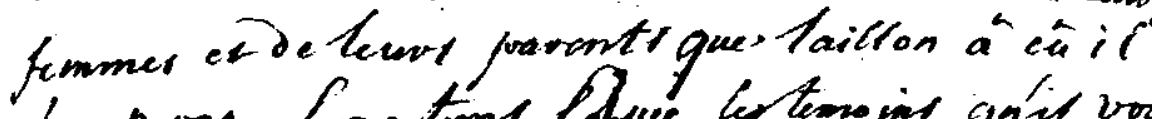

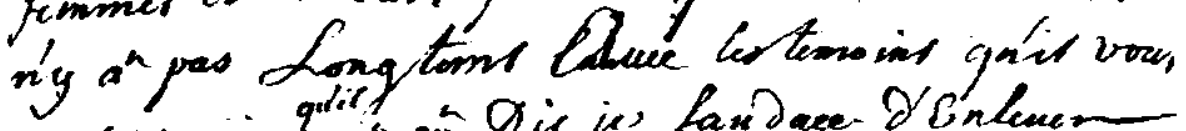

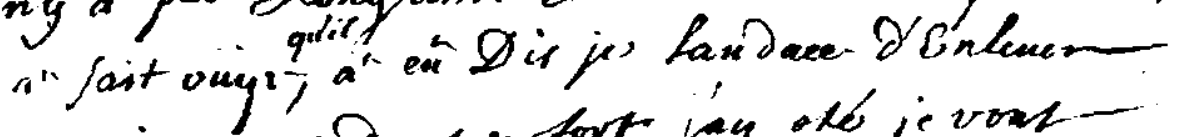
publiquement Dant ce forti jay ote je rout -

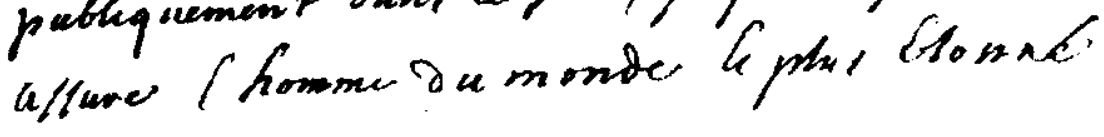




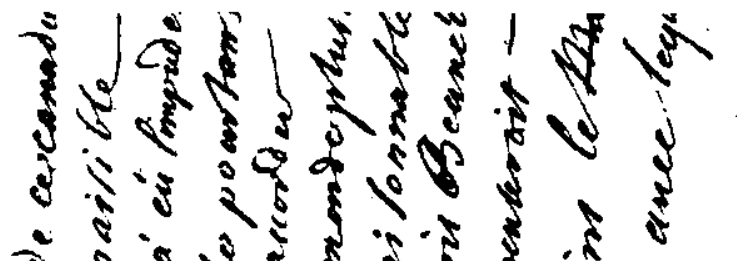

$\therefore$ कर

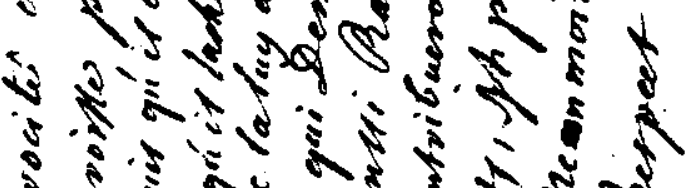

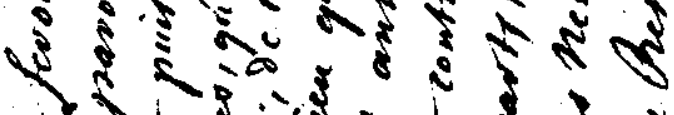
nos.

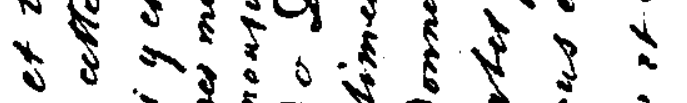

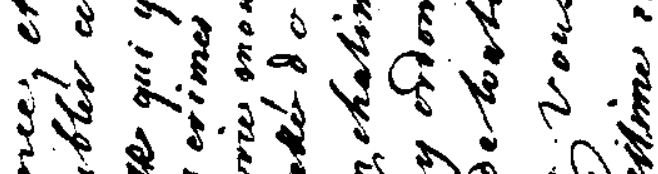

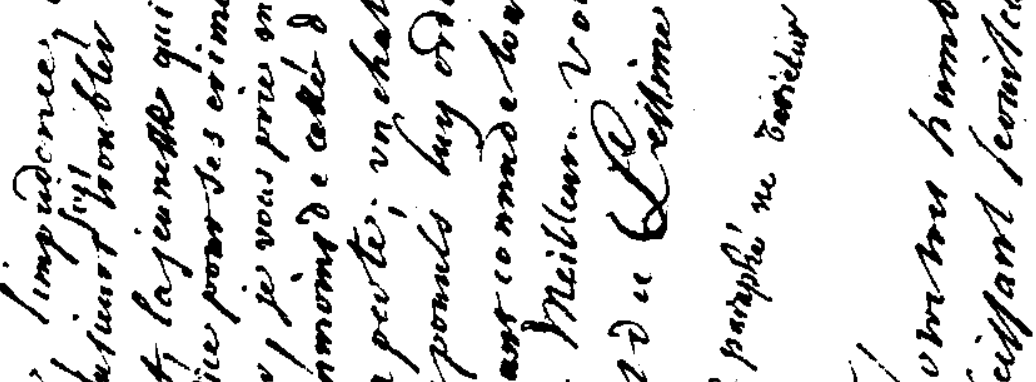

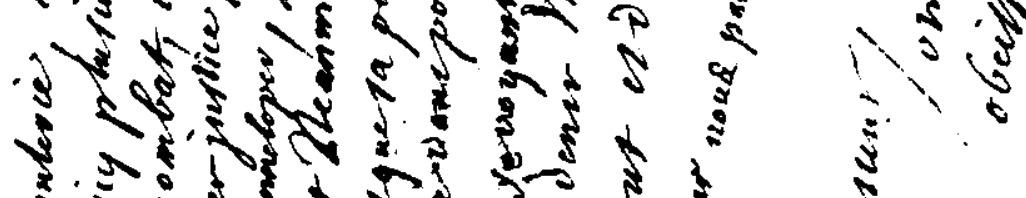

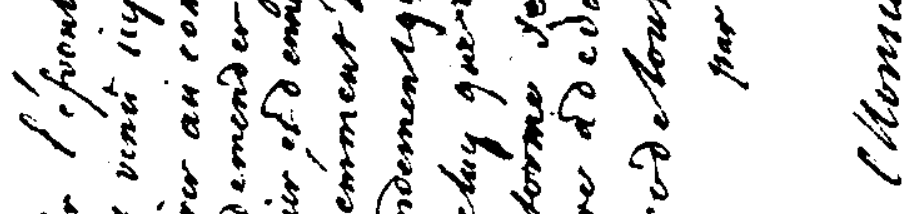

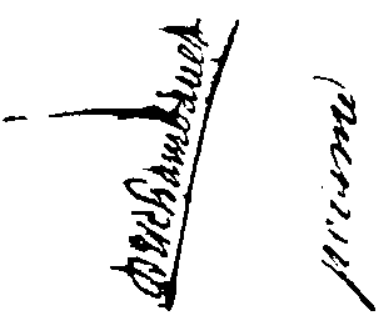

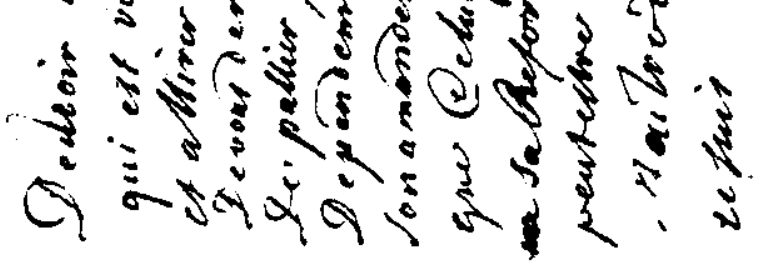


(Transcription de la lettre manuscrite dans une orthographe moderne mais où la ponctuation de l'auteur est respectée)

JMJ

À la Pointe-aux-Trembles le 23e juin 1708

\section{Monsieur,}

La charité et la sollicitude pastorale qui me rend très sensibles les affaires de mes paroissiens m'obligent de me donner l'honneur de vous écrire celui au sujet d'un nommé Taillon qui s'est mis entre les bras de la justice pour accabler deux de mes paroissiens que je reconnais pour honnêtes hommes, après avoir tenté par une malice inouie de les flétrir par sa langue ou de les opprimer par ses cabales factieuses. Ce que j'ai l'honneur de vous annoncer là, monsieur, je le tiens de tout un public pas un de ce fort et des environs ne m'a encore nié ce que hommes femmes et enfants m'ont d'abord dit du célèbre Taillon (voyez dans la ciincluse le portrait qu'en fait $\mathbf{M}$. De St.Come son curé) M. Genet m'a raconté la peinture qu'il vous en a faite, elle vous a surpris, et vous avez déclaré que vous chatieriez ce mutin s'il vous apportait des preuves convaincantes de ce qu'il avançait; je ne crois pas monsieur que vous récusiez celles-ci; et que vous me refusiez la grâce que j'ose vous demander fondé sur le bon droit en faveur des Beauchamps. Ce serait couronner le crime que d'écouter davantage le misérable Taillon au préjudice de ces honnêtes gens; qui ont voulu seulement soutenir l'honneur de leurs femmes et de leurs parents que Taillon a eu il n'y a pas longtemps avec les témoins qu'il vous a fait ouïr, qu'il a eu dis-je l'audace d'enlever publiquement dans ce fort; j'ai été je vous assure l'homme du monde le plus étonné de voir l'effronterie, l'impudence, et la férocité de ce Canadien qui est venu ici plusieurs fois troubler cette paroisse paisible et attirer au combat, la jeunesse qui y est puisqu'il a eu l'impudence de vous demander justice pour ses crimes mêmes, qu'il tâche pourtant de pallier et d'envelopper, je vous prie monsieur, de la lui accorder dépendemment néanmoins de celle de Dieu qui demande plutôt son amendement que sa perte; un châtiment aussi raisonnable que celui que vous pouvez lui ordonner contribuerait beaucoup à sa réforme, se voyant connu de 
toutes parts, il penserait peut-être à devenir meilleur. Vous êtes néanmoins le maitre de tout et de l'estime et du respect avec lequel je suis

monsieur

Votre très humble et très obéissant serviteur

Roche (?)

(Chaque page de cette lettre porte en outre une inscription du juge royal Jacques-Alexis Fleury Deschambault à qui elle était adressée)

"par nous paraphé ne varietur

ce $24^{\mathrm{e}}$ juillet 1708

Deschambault»

Note : Dans une autre lettre jointe au dossier, le curé St.Côme de l'île Jésus décrit Taillon comme «fort mutin, brouillon, brusque et emporté à l'excés et cherchant querelle à tout le monde».

(Source: Archives nationales du Québec, Montréal, dossier judiciaire du 20 juin 1708) 